Thompson prize for the most meritorious performance was awarded to R. G. Munro (of the Marconi Wireless Telegraph Co.). Most of the entries were of a very high standard, and the selection of the prize-winner must have presented a very difficult problem for the judges.

As usual, an exhibitors' meeting was held during the exhibition to discuss possible improvements in the organization of future exhibitions. The Council and Exhibition Committee of the Physical Society are to be congratulated on yet another successful exhibition.

A. B. Woon

\section{FUNCTION AND LOCATION OF ENZYMES IN CELLS}

A

T a meeting of the Royal Society on March 12 the subject of "The Function and Location of Enzymes in Cells" was discussed by a number of workers from Great Britain and abroad.

Prof. J. Z. Young (Department of Anatomy; University College, London), in opening the discussion, emphasized that the essence of the problem is to develop methods of high resolution. Much can be done by centrifugation and other methods of separation, but in the end we shall have to depend upon microscopy. Full use of microscopic methods would involve improvement of means of measurement, and a step in this direction might be found in the development of the technique of the flying-spot microscope. He also directed attention to the need for adequate models with which to speak about the organization of processes within the cells, and suggested that perhaps the techniques of information theory might be helpful to biochemists in this respect.

Dr. H. Holter (Carlsberg Laboratory, Copenhagen) described his experiments with the large amoba Chaos chaos and discussed the possibility of separation of different phases within its cytoplasm by centrifugation of the living cell. As test enzymes for these investigations, he chose succinic dehydrogenase, dipeptidase and catheptic proteinase, the last an enzyme likely to be difficult to localize because it is especially sensitive to autolytic changes, of which it is itself a mediator.

After cutting centrifuged amœbæ into heavy and light portions, it was found that their dipeptidase content was equal, which means that it is not associated with any centrifugable cell constituent but occurs throughout the cell in the hyaloplasm. On the other hand, the proteinase and succinic dehydrogenase were clearly displaced towards the centrifugal pole and are presumably bound to the mitochondria.

Comparable results were obtained by the technique of centrifugal separation after homogenization, except that the proteinase is liberated by this process from the cell constituent to which it is usually attached. Dr. Holter emphasized that homogenization techniques must, however, always give rise to equivocal results because the hyaloplasm is insoluble in water unless autolysis occurs.

Prof. J. F. Danielli (Department of Zoology, King's College, London) discussed the difficulties involved in the identification of the sites of phosphatase action by cytochemical techniques. He showed that phosphatase is found at sites concerned with active transport, such as the brush border of the proximal convoluted tubules of the kidney, epithelial cells of the small intestine and silk-secreting glands. He went on to develop the thesis that the enzyme is concerned particularly with making energy available for contractile proteins. Thus considerable amounts of phosphatase are found along chromosomes and during new collagen formation. During discussion of this paper, some objections to this hypothesis were raised by Prof. C. D. Darlington and others, who were doubtful whether the association of phosphatases with, for example, nucleoli, could be regarded as evidence of their action upon contractile proteins.

The distribution of enzymic activities of the mitochondria of pea seedlings were then discussed by Dr. D. D. Davies (Department of Botany, University College, Hull), who presented strong evidence that the enzymes responsible for the Krebs cycle in plants are closely associated with the mitochondrial fraction. Each step of the Krebs cycle was demonstrated by chromatographic identification of products in partially inhibited systems.

Dr. S. J. Holt (Courtauld Institute of Biochemistry, Middlesex Hospital Medical School, London) described the development of new staining techniques for the intracellular localization of enzymes, beginning with the necessity of finding readily penetrating chromogenic substrates of low molecular weight, but yielding highly insoluble products. The oxidation of indoxyl to indigo has been found to meet most requirements for a chromogenic reaction. The reactions of various substrates derived from indoxyl have been investigated and several found which are very suitable for the revelation of esterases. The chief problems are to obtain a precipitate of negligible granularity and to prevent diffusion effects. Using substrates such as 5-homoindoxyl acetate and 5 : 7-dichloroindoxyl acetate in the presence of suitable oxidizing agents, it is possible to show fine details of the distribution of esterases in motor end-plates and, for example, in the cells of the kidney, liver and pancreas. Methods for phosphateses have also been developed, and extension to other enzymes is well advanced. During discussion of this paper, it was suggested that false localizations might be obtained, due to the activity of oxidizing enzymes producing an indigo at their site of action. Dr. Holt replied that this had been fully investigated and that the same localizations were found in the presence of high concentrations of oxidase inhibitors.

Dr. F. R. Johnson (Department of Anatomy, University of Sheffield) presented slides, prepared by the Gomori technique, of the distribution of alkaline phosphatase in the epithelial cells of the small intestine. He showed that the appearance of the deposit of cobalt sulphide depends on control of the last steps of the technique and is related to the strength and age of the ammonium sulphide solution used. Under suitable conditions, a bilaminar distribution of the enzyme is seen in this tissue and also in kidney proximal convoluted tubules. Prof. H. A. Krebs pointed out that this distribution corresponds to that expected from suggested biochemical mechanisms of transport; but Prof. Danielli was doubtful whether the bilaminar appearance could be regarded as truly reflecting the distribution of the enzyme in the living cell.

Experiments showing that dipeptidase activity is a property of polynucleotides were described by Prof. F. Binkley (Emory University School of Dentistry, Georgia), who has been able to isolate a non-protein fraction from pig kidney which hydrolyses dipeptides such as cysteinylglycine. This activity is not 
destroyed or decreased by proteolytic enzymes, or in other procedures directed to remove all proteins, or by heating to $80^{\circ} \mathrm{C}$., or concentration by evaporation on a water-bath. Further evidence for the absence of protein was obtained by the negative result of standard protein tests and by the fact that the material did not induce antibody formation. Ultraviolet absorption spectra were typical of nucleic acids, and chromatographic analysis showed good correlation with the properties of polynucleotides. Prof. Binkley emphasized that there is no evidence to suggest that any dipeptidase is a protein, and no real surprise should be caused by the discovery that they may be something other than protein. Electrophoretically homogeneous preparations could be prepared by extraction with aqueous acetone and autolysis at $50^{\circ}$ C. Subsequent fractional precipitation with ethanol leaves the active component in the supernatant, and a crystalline product can be isolated of extremely high activity compared with that of proteolytic enzymes composed of protein. Some of the difficulties of this interpretation were mentioned, particularly that ribonuclease or snake venom does not destroy the enzyme activity, except at a low $p H$ at which activity is rapidly lost even without added enzymes. However, these enzymes do combine in $\mathbf{1}: \mathbf{1}$ proportions with the active material and inhibit it. A second difficulty is that commercial nucleic acids are not active as dipeptidases; but they may be made active by a purification procedure. Further experiments have shown that plant viruses have similar peptidase activities and that these are actually enhanced by digestion with proteolytic enzymes.

In the discussion of Prof. Binkley's paper, no convincing difficulties in his interpretation were raised. Prof. J. D. Bernal emphasized the extent to which our views on protein synthesis would be clarified if we could start with the presumption that nucleic acids were themselves proteolytic enzymes.

The localization of enzymes in muscle were discussed by Dr. S. V. Perry (School of Biochemistry, Cambridge), showing in particular that adenosine triphosphate cannot be free in the cell. The myofibrils probably contain bound adenosine triphosphate and the mitochondria an enzyme responsible for splitting it.

Prof. A. Claude (Laboratory of Cytology and Experimental Cancerology, University of Brussels) described his methods for separating cells into fractions of distinct particle size and enzymic activity. He showed that, even with small differences of density, sharp separation is possible by successive centrifugation, though this may be interfered with by agglutination. $\mathrm{He}$ compared quantitative determinations of the quantity of glycogen obtained by centrifugation and by chemical separation, and also clearly illustrated the value of this technique as a preparative method by showing gram samples of cell constituents isolated in a short working-time. Prof. Claude then surveyed the morphological features of cells and discussed the fine structure of mitochondria, as revealed by electron microscopy. In conclusion, he pointed out the need for developing more refined centrifugation techniques for the further separation of similar particles, such as mitochondria, into types with different enzymic activities.

Prof. H. A. Krebs (Department of Biochemistry, University of Sheffield) outlined experiments on the distribution of ions and inorganic substances in cells. $\mathrm{H} \theta$ emphasized that an understanding of these dis- tributions depends upon our treating the cell as not being in osmotic equilibrium, for the osmotic pressure in a cell may be two or three times that of the blood plasma. The conception that the cell is enveloped by a semi-permeable membrane is not complete because there are many intracellular boundaries; many substances may thus penetrate the cell and yet not gain access to all parts of it. Distribution of sodium ions is maintained by the mechanism of a 'sodium pump' actively removing the ion, and this may occur not only at the cell surface, but also at surfaces within the cell.

Experiments with slices of guinea pig brain and kidney cortex in which the potassium loss and sodium gain were studied were next described by Dr. R. E. Davies (Department of Biochemistry, University of Sheffield). Under proper conditions reversible interchanges can be recorded. The water content of the tissue depends more on its metabolic activity than on the composition of the medium. Thus the turnover number of sodium ions to oxygen consumed was very high $(5,000: 1)$, and it is necessary to postulate an energy-linking system between entry to and escape from the cell. Treatment with dinitrophenol unlinks the process of transport from its energy sources and allows accumulation of sodium within the cell. The whole of the sodium and potassium content of a cell was found to be able to exchange in a very short time. Dr. Davies then presented evidence that these activities are controlled by enzymes located in the mitochondria. Fractions consisting mainly of mitochondria are thus able to concentrate ions and pump out water.

In elosing the discussion, Prof. Young thanked the contributors for the various original observations reported during the day; he directed attention to the fact that the only mention of a new model was Prof. Krebs's comparison of the cell with a leaky ship kept afloat by pumps.

\section{SAMPLE TABLES FOR THE 195। CENSUS OF GREAT BRITAIN}

TN the past, the General Register Office has often been severely criticized for its delay in the publication of the results of the decennial census of population. The critics often ignored the fact that the professional staff of the Office was very small, and that they had to cope with the routine work of the Office in addition to the census. It is therefore most creditable to the Office that a summary of the main results of the 1951 census came into the hands of the public within nineteen months of the date of enumeration*.

This was only made possible by the decision-for the first time in the history of censuses in Great Britain - to make a preliminary analysis of a sample of 1 per cent of the census schedules. Sampling had an additional advantage - tabulations which would have been prohibitively expensive and complicated in a full census were quite feasible for a sample. A sample of census schedules is also likely to be more accurate than an ad hoc inquiry : the completion of the schedule is compulsory, and sample selection can therefore be strictly controlled. The problem of non-response is minimized, and there is probably

* General Register Office and General Registry Office Scotland. Census 1951, Great Britain : One Per Cent Sample Tables. Pp. 367. (London: H.M.S.O., 1952.) Part 1, 178. 6d.; Part 2, £2. 